\title{
Influence of psychological profile in autonomic response of nursing students in their first hospital clinical stays
}

Paula Sánchez-Condea; Ana Isabel Beltrán-Velascoa; Vicente Javier Clemente-Suárez

\begin{abstract}
Background: This research aimed to analyse the psychological profile of nursing students and its relationship with the physiological anticipatory anxiety response at the earliest clinical practices; and to analyse the habituation response at the psychophysiological level of nursing students throughout their first clinical practices. Methods: 18 students of the first year of Nursing degree were studied in their first clinical stays. Their psychophysiological stress response was evaluated throughout the analysis of the autonomic modulation and their subjective perception to stress at the beginning and at the end of the stays. In addition, an inventory of psychological test was carried out to observe its association with the psychophysiological stress response. Results: The results showed that the students presented a large anticipatory anxiety response at the beginning of the stays, as well as an absence of a habituation process during the stay. It was also observed a positive relation between the loneliness perceived and the autonomic stress response. Conclusion: The clinical stays in the hospital performed by first year students of the nursing degree, did not produce the expected habituation response at psychophysiological level. Also, a higher perception of social isolation was significantly related with a higher objective (sympathetic modulation) and subjective (perceived stress) stress responses.
\end{abstract}

Keywords: Stress, Personality, Social isolation, Profile, Nursing 